\title{
Measurement of radius of a metallic ball using eddy current testing based on peak frequency difference feature
}

\author{
Gang $\mathrm{Hu}^{1}$, Ruochen Huang ${ }^{1}$, Mingyang $\mathrm{Lu}^{1}$, Lei Zhou ${ }^{2}$, Wuliang Yin ${ }^{1}$
}

\begin{abstract}
This paper proposes a linear eddy-current feature to determine the radius of a metallic ball in a non-contact manner. An electromagnetic eddy-current sensor with two coils is placed co-axially to the metal ball during measurement. It is well known that the distance between the sensor and test piece (i.e. lift-off) affects eddy-current signals. In this paper, it is found that the peak frequency feature of inductance spectrum is linear to the lift-off spacing between the centre of coil and ball. Besides, the slope of peak frequencies versus lift-offs is linked to the radius of ball. The radius of metallic balls is retrieved from the experimental and embedded analytical result of the slope. Measurements have been carried out on 6 metallic balls with different radii. The radius of the metallic ball can be retrieved with an error of less than $2 \%$.
\end{abstract}

Index Terms - Eddy current sensor, lift-off variation, radius measurement, peak frequency feature, multi-frequency testing

\section{INTRODUCTION}

Conductive spherical balls are widely used in bearings applicable in robots, aircraft, automotive, and medical devices, for example, the spherical joint is used to support the flexible movements of the robots. Conductive spherical surfaces are used in screen EM interferences or used in certain containers/tanks for gas/oil storage/transportation. Hip replacement joint is an example of spherical surfaces in medical applications. Grinding balls are widely used in heavy industries. Moreover, the conductive coatings of the sphere object can also be estimated to ensure safety following this line of work. In addition, spherical balls are convenient to serve as calibration samples for material characterisation, in particular when the available sample size is limited. Normally, when using plate / cylindrical samples, there is a requirement that their sizes (planar dimension of a plate / height of cylindrical samples) should be significantly larger than the sensor coil dimension to eliminate edge effect.

Eddy-current (EC) is one of the most popular approaches exploited to inspect conductive materials. It is commonly used to assess the thickness of metal coating, to inspect the integrity of metal structures, to determine electrical conductivity and magnetic permeability of the material due to high sensitivity and good adaptability to tested objects [1-5]. This has become one of the main methods in non-destructive testing techniques. It is capable of testing the samples without contact with the samples while maintaining high precision and performance. As

1 School of Electrical and Electronic Engineering, University of Manchester, Oxford Road, Manchester, M13 9PL, UK

Corresponding author: $\quad \underline{\text { ruochen.huang@manchester.ac.uk; }}$ the eddy current is measured, the alternating current is fed into the excitation coil to produce alternating magnetic fields and then induce eddy current in conductive samples. Thus, the transmitting coil receives a signal produced from the magnetic field produced from the exciting coil and the reflected signal from the sample. There are mainly two methods in the area of eddy current testing depending on the form of the excitation signal, i.e. pulsed eddy current (PEC), and multi-frequency eddy current (MEC). Multi-frequency eddy current testing has become a reliable technique for on-line and off-line measurements in our previous work. However, lift-off has a significant effect on the measurement's accuracy for either surface crack detection or material properties (conductivity, magnetic permeability, radius, thickness, etc.) measurements[6]-[11].

For certain cases, the examined objects can have a spherical surface and the radius of the sample may be determined by EC [12].

Several techniques have been proposed to address the issues caused by the lift-off effect. A hypothetical transformation model was applied in the PEC technique shows it effects in reducing the lift-off effect for planar geometry [13]. A new triple electromagnetic helix sensor was developed by Yin et al. and measurements were found to be resistant to lift-off variations [14]. Yin et al. showed that the phase signature is less sensitive to the lift-off of the non-magnetic metal plate compared with magnitude [15]. In addition, a compensation algorithm was developed by $\mathrm{Lu}$ et al. for reducing lift-off in thickness measurements for metallic plates. [16]. Yang et al used a differential PEC probe and FFT transformation to classify the depth of the defect. It is found that the lift-off effect can be reduced under the lower band of frequency spectral and the depth can be extracted according to the peak amplitude [9]. This feature is further exploited in[17]. Abu-Nabah reduced the lift-off effect by using harmonic eddy current excitation and a GMR sensor configuration [18]. Other methods of using PEC and MEC is suggested to reduce the lift-off effect of the when measuring the thickness of the metallic film [20]-[21].

Our group have proposed the peak frequency, the zerocrossing, and the phase feature in the attempt to reduce the liftoff effect on sample property measurements [22]-[33]. A novel algorithm was proposed to solve the lift-off problem by estimating the thickness of metallic film based on the highfrequency characteristics of the sensor response [34]. A lift-off- 
tolerant pancake eddy current sensor was devised for the sensor to work at a larger range of lift-offs [35]. Another way to remove the lift-off effect is to work out the lift-off itself. When measuring at high frequency, eddy current thin skin effect can be exploited and a method based on the phase feature of the material can be used to achieve high accuracy [36]. The dualfrequency lift-off linearity, particularly at higher operating dual frequencies can be used to increase lift-off tolerance to 10 $\mathrm{mm}[37]$. In addition to our previous work, the lift-off and sample parameters are explicitly retrieved from high-frequency features. However, these techniques only refer to the sample geometry of thin foils or slabs.

In this paper, the peak frequencies are found linear to the liftoff distance between the centre of coil and non-ferrous metal ball. With the capability of this method proposed, the integrity and quality of the objects in the examples mentioned can be tested in non-destructive and non-contact manner. Based on the peak frequency difference from two lift-offs, it can be applied to estimate spherical surfaces. For objects with plane surfaces, some investigations have been carried out to eliminate the liftoff effect, as reported in papers.[14][38]-[39]. Besides, the radius of metallic ball is retrieved by referring to the slope of peak-frequency versus lift-offs. Measurements have been carried out on six metal balls with different radii. The radius of metallic ball can be retrieved with an error of less than $2 \%$.

\section{LINEARITY FEATURES AND RADIUS CALCULATION ALGORITHM}

Fig. 1 shows the geometry of the air-cored EM sensor. The excitation coil and the receive coil are located co-axially above the spherical sample. These two coils have identical properties, i.e. size, turns and material.

\section{A. Structure of the sensor}

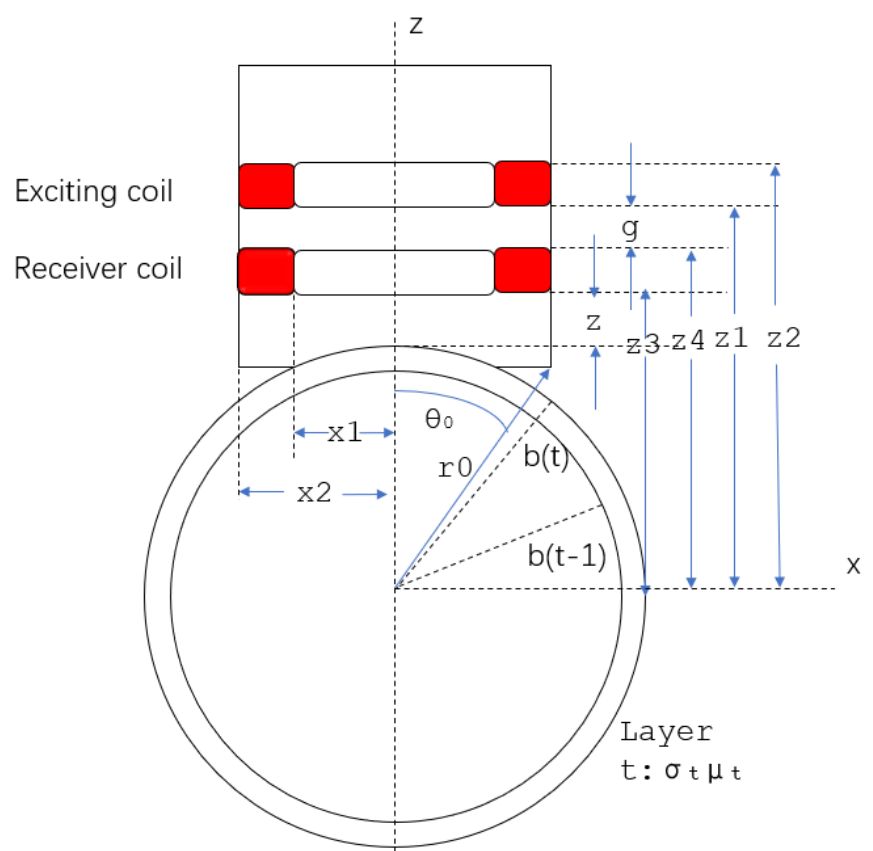

Fig. 1 Sensor structure
TABLE I COIL PARAMETERS

\begin{tabular}{|c|c|}
\hline Inner radius $\left(\mathrm{x}_{1}\right)$ & $17.5 \mathrm{~mm}$ \\
\hline Outer radius $\left(\mathrm{x}_{2}\right)$ & $17.9 \mathrm{~mm}$ \\
\hline The coil separation (g) & $5.0 \mathrm{~mm}$ \\
\hline $\begin{array}{l}\text { The distance between the centre of the } \\
\text { spherical sample and the lower/upper } \\
\text { height of the excitation coil }\left(z_{1} / z_{2}\right)\end{array}$ & $65.3 \mathrm{~mm} / 71.3 \mathrm{~mm}$ \\
\hline $\begin{array}{l}\text { The distance between the centre of the } \\
\text { spherical sample and the lower/upper } \\
\text { height of the receive coil }\left(\mathrm{z}_{3} / z_{4}\right)\end{array}$ & $52.3 \mathrm{~mm} / 60.3 \mathrm{~mm}$ \\
\hline $\begin{array}{l}\text { The distance between the sphere } \\
\text { vertex and the lower height of the } \\
\text { receive coil (z) }\end{array}$ & $2.0 \mathrm{~mm}$ \\
\hline The radius of the spherical sample $\left(b_{N}\right)$ & $140 \mathrm{~mm}$ or $160 \mathrm{~mm}$ \\
\hline Turns $\left(N_{1} / N_{2}\right)$ & $20 / 20$ \\
\hline
\end{tabular}

For the sensor setup, $r_{0}$ and $\theta_{0}$ need to be mentioned particularly. Here $r_{0}$ denotes radial distance, and $\theta_{0}$ denotes latitude. These two parameters can used to locate the coil at space. The value of each parameter is shown in the table I. The conductivity value of each simulated metal is listed in table II.

TABLE II METAL CONDUCTIVITY

\begin{tabular}{ll}
\hline \hline Material & $\begin{array}{l}\text { electrical conductivity } \\
(\mathrm{MS} / \mathrm{m})\end{array}$ \\
\hline Copper & 58.00 \\
Brass & 25.00 \\
Aluminium & 36.00 \\
Zinc & 17.40 \\
Titanium alloy & 0.59 \\
Stainless steel & 1.38 \\
\hline
\end{tabular}

\section{B. Analytical formulation}

The inductance change of the air-cored sensor coil caused by the nonmagnetic, metallic spherical sample has been proposed [40]. The coil impedance $Z$ can be represented by the sum of the coil impedance in free space $Z_{0}$ and the impedance change induced by the eddy currents within the spherical sample $\Delta Z$.

$$
\mathrm{Z}=\mathrm{Z}_{0}+\mathrm{D} \boldsymbol{Z}
$$

As shown in equations (2)-(4), due to the phase difference between the voltage and current, the measured impedance between the excitation coil and receiving coil should be complex value, contains real part and imaginary part. The inductance change is defined as the ratio of the impedance change and the excitation frequency. The imaginary part of the inductance change reflects some properties of resistive components.

$$
\begin{gathered}
\Delta \mathrm{Z}=\Delta \mathrm{R}+\Delta \mathrm{jX} \\
\operatorname{Re}(\Delta \mathrm{L})=\operatorname{real}\left(\frac{\Delta \mathrm{Z}(\mathrm{f})}{\mathrm{j} \omega}\right)=\frac{\Delta \mathrm{X}}{\omega} \\
\operatorname{Im}(\Delta \mathrm{L})=\operatorname{imag}\left(\frac{\Delta \mathrm{Z}(\mathrm{f})}{\mathrm{j} \omega}\right)=\frac{\Delta \mathrm{R}}{\omega}
\end{gathered}
$$


Where $Z(f)$ represents the impedance of the coil in the presence of a metallic ball and $Z_{0}(f)$ is that of the coil in air.

Due to the presence of the spherical sample, the impedance change can be expressed as,

$$
\begin{gathered}
\Delta Z=\frac{j \pi \omega N_{1} N_{2}}{\left(z_{2}-z_{1}\right)\left(z_{4}-z_{3}\right)\left(x_{2}-x_{1}\right)\left(x_{4}-x_{3}\right)} * \\
\sum_{n=1}^{\infty} \frac{b_{t}^{2 n+1}}{n(n+1)} \frac{V_{11 N} N O M I+V_{21 N} N O M K}{V_{11 N} D E N I+V_{21 N} D E N K} P_{1, n, S} P_{2, n, s}
\end{gathered}
$$

Where

$$
\begin{array}{r}
N O M I=i_{n}\left(a_{N} b_{N}\right)\left[(n+1) \mu_{N}-1\right]-a_{N} b_{N} i_{n}^{\prime}\left(a_{N} b_{N}\right) \\
N O M K=k_{n}\left(a_{N} b_{N}\right)\left[(n+1) \mu_{N}-1\right]-a_{N} b_{N} k_{n}^{\prime}\left(a_{N} b_{N}\right) \\
D E N I=i_{n}\left(a_{N} b_{N}\right)\left(n \mu_{N}+1\right)+a_{N} b_{N} i_{n}^{\prime}\left(a_{N} b_{N}\right) \\
D E N K=k_{n}\left(a_{N} b_{N}\right)\left(n \mu_{N}+1\right)+a_{N} b_{N} k_{n}^{\prime}\left(a_{N} b_{N}\right) \\
a_{\mathrm{t}}=\sqrt{j \omega \mu_{t} \mu_{0} \sigma_{t}} \\
P_{n, S}=\int_{\theta_{21}}^{\theta_{12}} \sin \theta_{0} P_{n}^{1}\left(\cos \theta_{0}\right) \int_{o 2}^{r_{o}\left(\theta_{o}\right)} \frac{1}{r_{o 1}\left(\theta_{o}\right)} d r_{0}^{n-1} d \theta_{0} \\
V(N)=T(N, N-1) T(N-1, N-2) \ldots T(2,1) \\
T(t+1, t)=\left[\begin{array}{ll}
T_{11}(t+1, t) & T_{12}(t+1, t) \\
T_{21}(t+1, t) & T_{22}(t+1, t)
\end{array}\right]
\end{array}
$$

Here equation (10) is related to the skin depth. The formula of skin depth is:

$$
\delta=\frac{1}{\sqrt{\pi f_{0} \mu_{t} \mu_{0} \sigma_{t}}}=\frac{\sqrt{2 j}}{a_{t}}
$$

Other parameters, $\mathrm{N}$ denotes the total shell number of the multi-layered spherical sample. The $t^{\text {th }}$ shell has a constant conductivity $\sigma_{t}$ and the relative permeability $\mu_{t} ; i_{n}\left(a_{N} b_{N}\right)$ and $k_{n}\left(a_{N} b_{N}\right)$ denote the modified spherical Bessel function of the first and second kind respectively; $i_{n}^{\prime}\left(a_{N} b_{N}\right)$ and $k_{n}^{\prime}\left(a_{N} b_{N}\right)$ denote the derivatives of the respective modified spherical Bessel functions. The interface between shells $t$ and $t+1$ is located at a radial distance $b_{t} ; \omega$ denotes the excitation frequency; $\mu_{0}$ denotes the permeability of free space; $\mu_{N}$ denotes the permeability of outermost spherical shell; $P_{n}^{1}(x)$ denotes the first order of Legendre function; $\mathrm{V}(\mathrm{N})$ is a twodimensional matrix and $V_{i j N}$ denotes the element in the $i^{\text {th }}$ row and $j^{\text {th }}$ column. The expressions of $\mathrm{T}$ matrix for different cases are introduced in the appendix.

As shown in equation (11), $P_{n, S}$ contains double integration considering the rectangular cross-section of the coil. It can be substituted with a single integration loop, which gives $n \neq 2$ :

$$
\begin{aligned}
& P_{n, S}=\int_{\theta_{12}}^{\theta_{11}} \frac{\sin \theta_{0} P_{n}^{1}\left(\cos \theta_{0}\right)}{n-2}\left(\frac{\sin \theta_{0}}{x_{1}}\right)^{n-2} d \theta_{0} \\
& +\int_{\theta_{11}}^{\theta_{21}} \frac{\sin \theta_{0} P_{n}^{1}\left(\cos \theta_{0}\right)}{n-2}\left(\frac{\cos \theta_{0}}{z_{1}}\right)^{n-2} d \theta_{0} \\
& +\int_{\theta_{21}}^{\theta_{22}} \frac{\sin \theta_{0} P_{n}^{1}\left(\cos \theta_{0}\right)}{n-2}\left(\frac{\sin \theta_{0}}{x_{2}}\right)^{n-2} d \theta_{0} \\
& +\int_{\theta_{22}}^{\theta_{12}} \frac{\sin \theta_{0} P_{n}^{1}\left(\cos \theta_{0}\right)}{n-2}\left(\frac{\cos \theta_{0}}{z_{2}}\right)^{n-2} d \theta_{0}
\end{aligned}
$$

$n=2$ :

$$
\begin{aligned}
& P_{n, S}=\int_{\theta_{12}}^{\theta_{11}} \sin \theta_{0} P_{n}^{1}\left(\cos \theta_{0}\right) \operatorname{In} \frac{x_{1}}{\sin \theta_{0}} d \theta_{0} \\
& +\int_{\theta_{11}}^{\theta_{21}} \sin \theta_{0} P_{n}^{1}\left(\cos \theta_{0}\right) \operatorname{In} \frac{z_{1}}{\cos \theta_{0}} d \theta_{0} \\
& +\int_{\theta_{21}}^{\theta_{22}} \sin \theta_{0} P_{n}^{1}\left(\cos \theta_{0}\right) \operatorname{In} \frac{x_{2}}{\sin \theta_{0}} d \theta_{0} \\
& \quad+\int_{\theta_{22}}^{\theta_{12}} \sin \theta_{0} P_{n}^{1}\left(\cos \theta_{0}\right) \operatorname{In} \frac{z_{2}}{\cos \theta_{0}} d \theta_{0}
\end{aligned}
$$

Where: $\theta_{i j}=\arctan \left(x_{i} / z_{j}\right)$. It denotes four angles of the rectangle corners. This parameter is mainly used to transfer double integral to a 1-D integral.

In the free space, the impedance $Z_{0}(f)$ can be calculated as

$$
\begin{aligned}
& Z_{o}=j X_{o} \\
& =\frac{j 2 \pi \omega \mu_{0} N_{S}^{2}}{\left(z_{2}-z_{1}\right)\left(z_{4}-z_{3}\right)\left(x_{2}-x_{1}\right)\left(x_{4}-x_{3}\right)} \int_{0}^{\infty} \frac{1}{a^{6}} J^{2}\left(x_{2}, x_{1}\right)\left[a\left(z_{2}-z_{1}\right)+e^{-a\left(z_{2}-z_{1}\right)}-1\right] d a
\end{aligned}
$$

where

$$
J\left(x_{2}, x_{1}\right)=\int_{a x_{1}}^{a x_{2}} x J_{1}(x) d x
$$

Here $J_{1}^{(x)}$ is the Bessel function of the first kind and first order.

Further, after obtaining the impedance change due to the spherical sample, the peak frequency can be extracted by searching the minimum value of the imaginary part from the ratio of impedance change and the excitation frequency.

$$
a=\min \{\operatorname{Im}((\Delta Z(f)) / j \omega)\}
$$

Where, a represents the peak frequency where the local minimum of the imaginary part of the inductance change is located. In order to calculate the peak frequency, it is found that, in a specific frequency range, the curve of imaginary part of the inductance change can be approximated as a quadratic function. The peak frequency can be calculated by finding the stationary point from the derivatives of the quadratic function.

\section{Linearity feature}

In this section, different setups (materials, radii and thicknesses) of sphere balls have been simulated. The metal ball is a double layered sphere (i.e. it is a hollow sphere: the inner sphere is made of air/ the shell is made of metal), the value of thickness of metal ball is the difference between the inner radius and the out radius. It can be seen from Figs. 2-4 that for different materials, radii and thicknesses, the peak frequency in (16) is 
linear to the lift-off distance. For metal balls with different radii, the slope of the peak frequency will decrease as the radius increases. For metal balls of different materials, the slope of the peak frequency changes more obviously, and it will decrease as the electrical conductivity of the material increases.

Fig. 5 presents the relationship between conductivity and slope of peak frequency versus lift-offs. It can be noted that, for different kinds of metals, as the conductivity increases, the value of the slope of the peak frequency versus lift-offs decreases. A slope can be worked out from the relationship between the peak frequency and lift-off, i.e., two peak frequencies can be obtained from two lift-offs, then a slope can be calculated. For spheres with different conductivity, the slope is different. Figure 5 illustrates the relationship between conductivity and the slope. It is obvious that the conductivity is inversely related to the slope of peak frequency. The conductivity of copper is $58 \mathrm{MS} / \mathrm{m}$ and the slope rate is approximately 5 , whereas the titanium metallic ball has conductivity of $0.58 \mathrm{MS} / \mathrm{m}$ and highest slope. Besides, the slope of the peak frequency will decrease as the thickness of the metal ball increases. Based on this, the slope of the peak frequency can be used to detect the material, radius, and thickness of the metal ball.

Therefore, based on the linear relationship of peak frequency with lift-offs, the inverse radius calculation can be deduced by using the slope rate, shown in Fig. 6 which describes the calculation process of obtaining the radius of the metallic ball. The first step is to measure the peak frequency at two lift-offs. Then, the slop rate can be calculated by using two differences $(\Delta \mathrm{Z}$ and $\Delta \mathrm{f})$ ), shown in the second and third step of the process chart. Consequently, by comparing the measured slope rate with the theoretical ones, the radius of ball can be obtained.

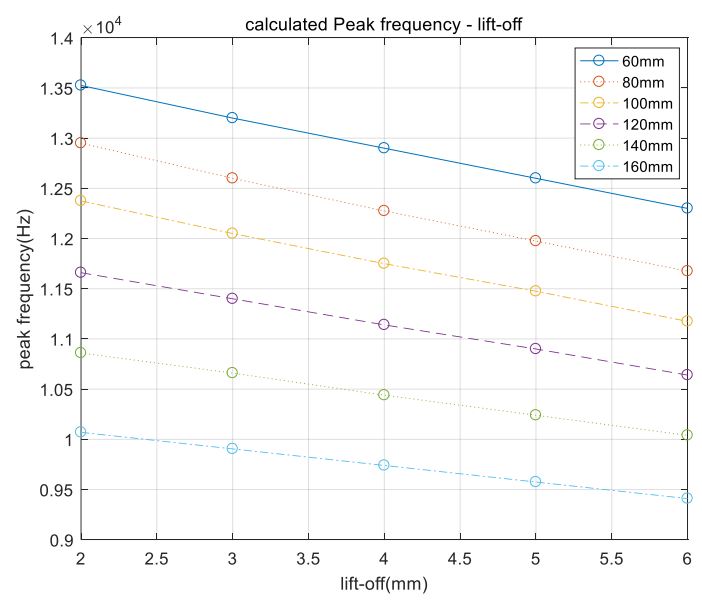

Fig.2. Comparisons of theoretical peak frequencies for the different size of stainless-steel ball at a range of lift-offs

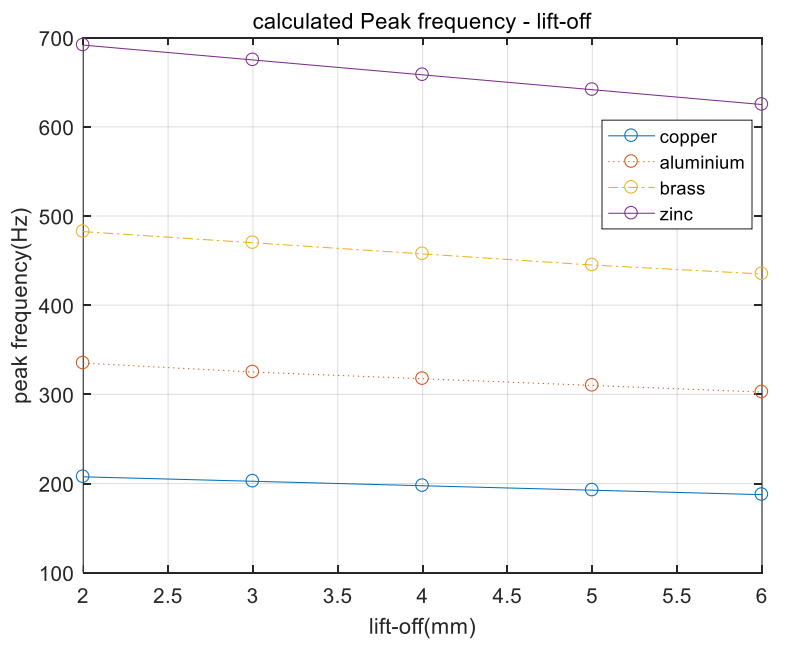

Fig.3 Comparisons of theoretical peak frequencies for the different balls of different materials at a range of lift-offs(thickness is $1 \mathrm{~mm}$ and radius is $160 \mathrm{~mm}$ )

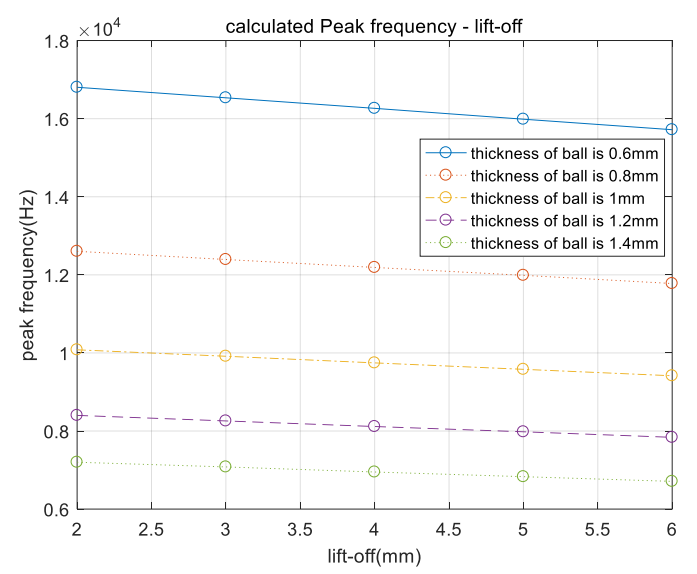

Fig.4. Comparisons of theoretical peak frequencies for the different thickness of stainless steel ball at a range of lift-offs(the radius is $160 \mathrm{~mm}$ )

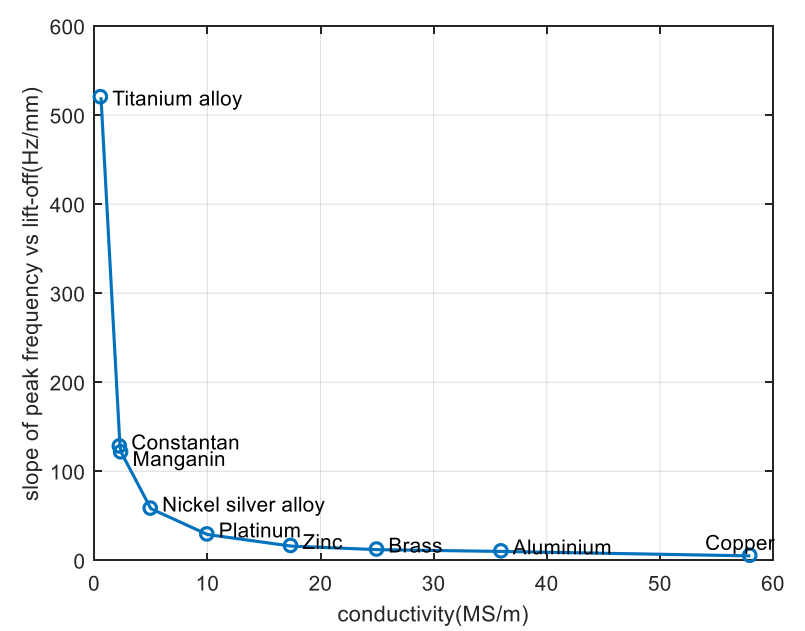

Fig.5. The relationship between conductivity and slope of peak frequency versus lift-off. 


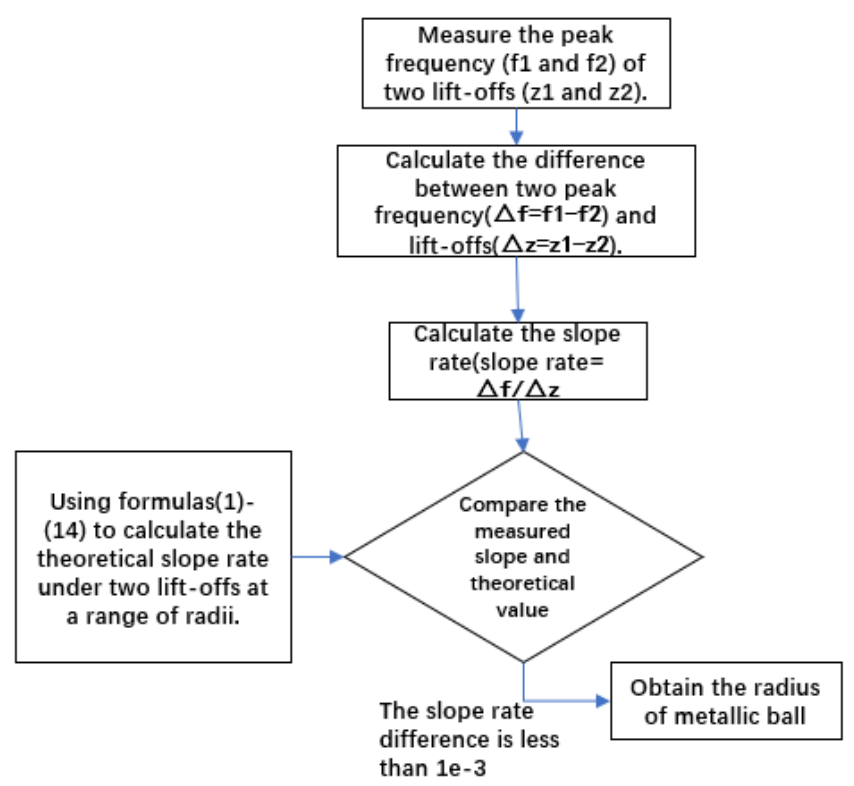

Fig.6 The calculating process of obtaining the radius of metallic ball

\section{EXPERIMENTS AND RESULTS}

\section{A. Experiment and simulation setup}

To evaluate the radius calculation algorithm due to the lift-off variation based on the linearity feature of peak frequency versus lift-offs, simulations and experiments have been carried out on 6 metallic balls with different radii.

The sensor configuration in experiments and simulations is listed in Table I. The simulations were programmed in MATLAB. The Zurich impedance analyser with frequency sweeping mode is applied to obtain the experimental data during the measurements. The experimental schematic diagram is shown in Fig.7. The spherical samples are stainless steel balls with the conductivity of $1.37 \mathrm{MS} / \mathrm{m}$ and the thickness of $1 \mathrm{~mm}$. As shown in Fig. 8, from the simulation, the peak frequency is no longer linearly decreasing when lift-off distance is larger than $30 \mathrm{~mm}$. Besides, in practical measurement, the sensor probe is placed close to the tested object to obtain a better performance. Therefore, the lift-off is chosen from $2 \mathrm{~mm}$ to 6 $\mathrm{mm}$ in the steps of $1 \mathrm{~mm}$.

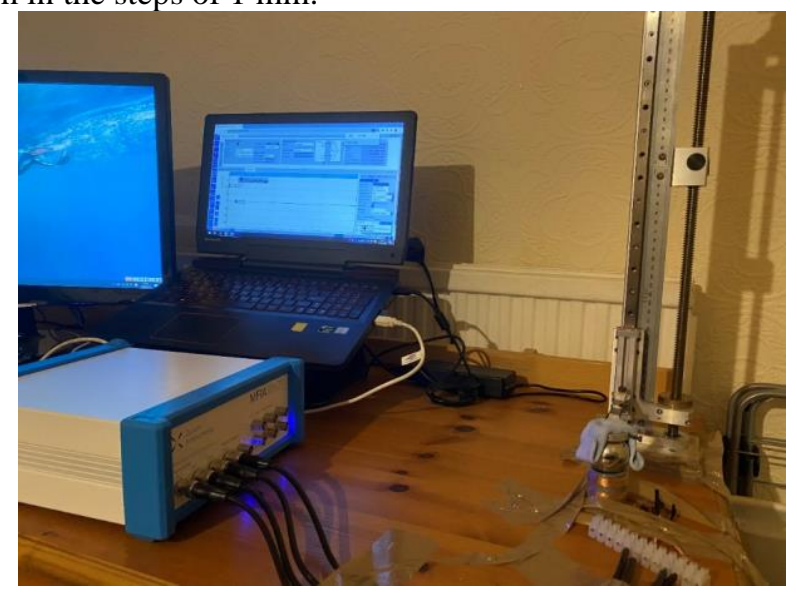

Fig. 7. The impedance analyser for the eddy current testing of metallic ball

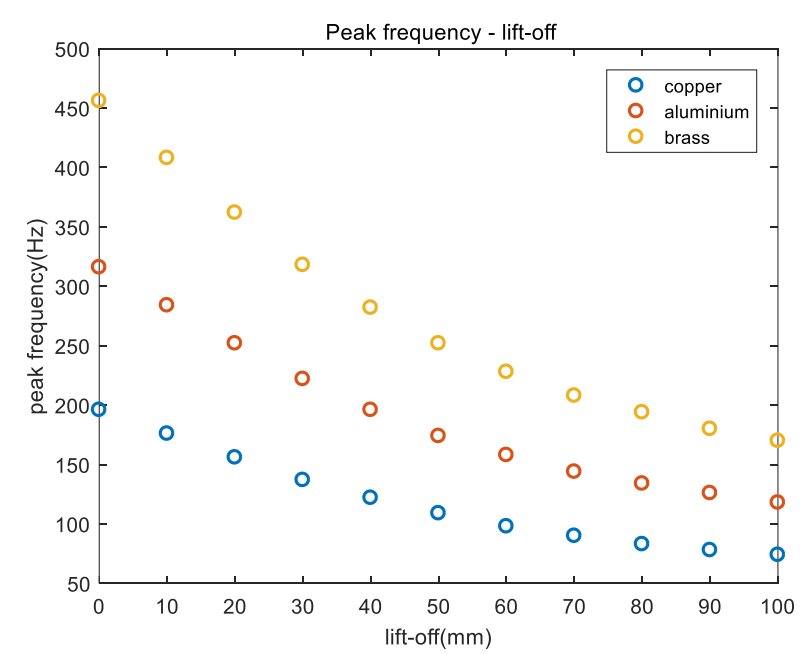

Fig. 8. Peak frequency of different metal in different lift-off distance

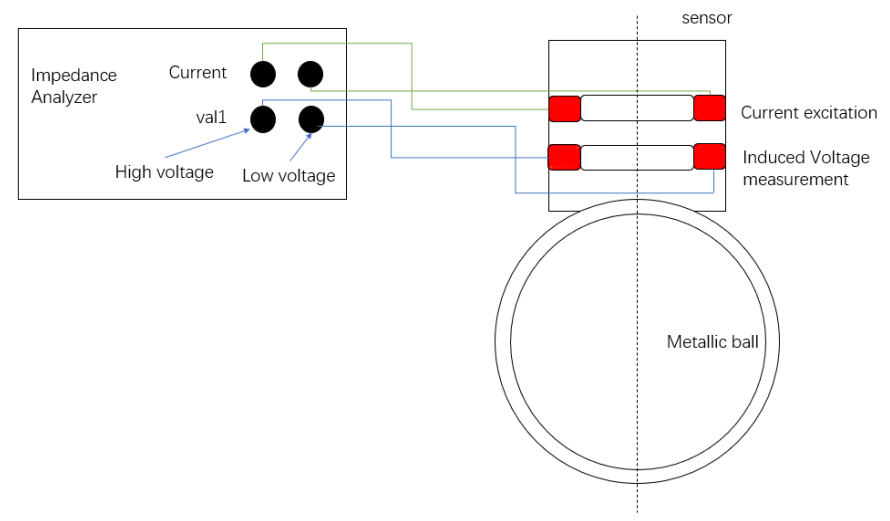

Fig.9. Experimental schematic diagram

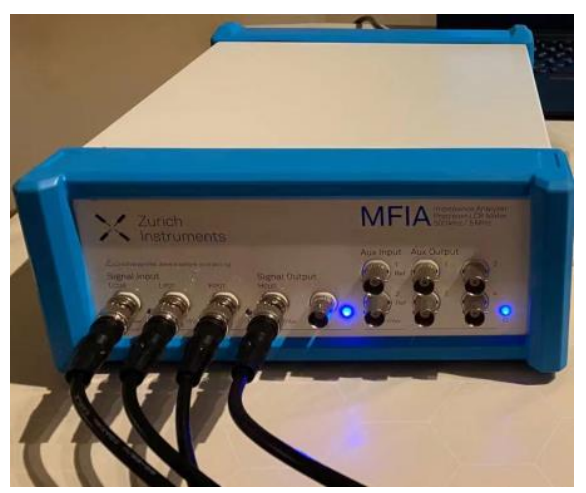

Fig.10. Interface of impedance analyser

\section{B. Results}

As shown in Fig. 11 and Fig. 12, for the coil-sample system, both the real and imaginary parts of the inductance change was measured under the multi-frequency spectra and the lift-off spacer was from $2 \mathrm{~mm}$ and $6 \mathrm{~mm}$. Experimental curves agree with simulation results. From both sets of results, it is found that the magnitude of both real part and imaginary part of the inductance change decreases as the lift-off increases. Besides, there exists a minimum value in the imaginary part and the 
corresponding frequency (named as peak frequency) is used to investigate the effect due to the lift-off. It can be seen that the shift of the peak frequency is not severe. The difference of peak frequency from the lift-off of $2 \mathrm{~mm}$ and $6 \mathrm{~mm}$ is only $\sim 1 \mathrm{kHz}$. Fig. 13 illustrates the inversely proportional trend between the peak frequency and the lift-off under different radii of metallic balls. Besides, due to more interaction between the magnetic field and the spherical ball, the peak frequency decreases as the radius increases. There is a slight difference in the peak frequency between the simulations and experiments which is due to the estimation of the peak frequency from the limited number of the frequency number. Moreover, the slope of metallic spherical sample increases with larger radius. By utilizing this feature, the radius of the metallic sphere can be deduced from different slopes shown in Fig. 13.

In measurements, the slope of the tested sample can be calculated by the two lift-offs testing. Then the inversion tool is used to match the simulation with the experiment. Therefore, the radius of the spherical sample can be estimated, and it proves to be robust with a reasonable variation of $2 \%$.

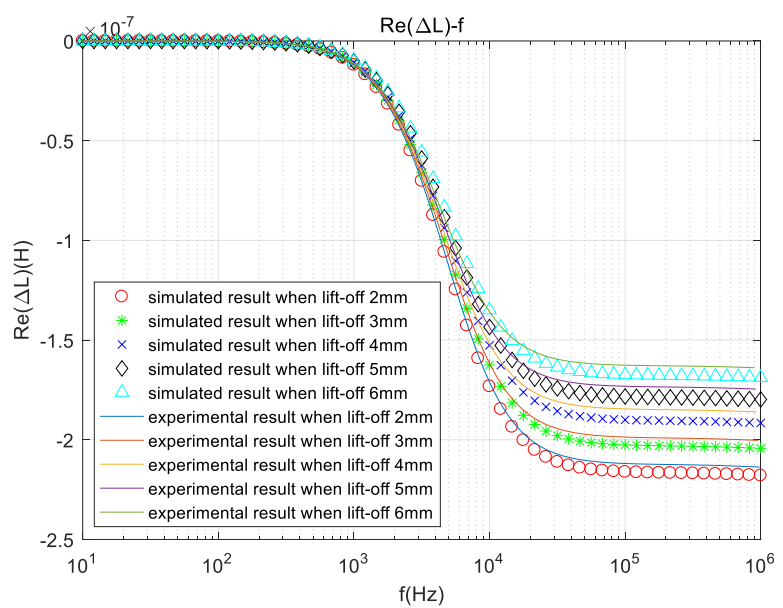

Fig. 11. Simulation and experimental results of real part of $\Delta \mathrm{L}$ for stainless ball with radius of $160 \mathrm{~mm}$ and thickness of $1-\mathrm{mm}$ at a range of lift-offs

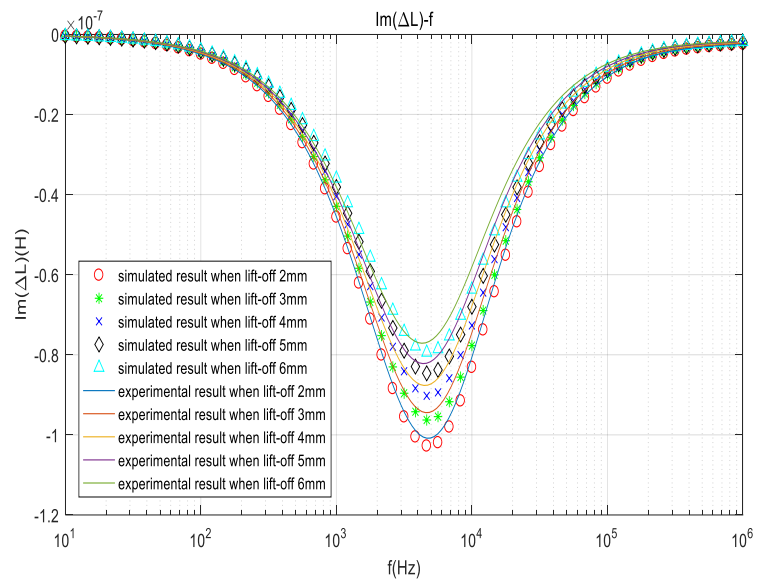

Fig. 12. Simulation and experimental results of imaginary part of $\Delta \mathrm{L}$ for stainless ball with radius of $160 \mathrm{~mm}$ and thickness of 1-mm at a range of lift-offs

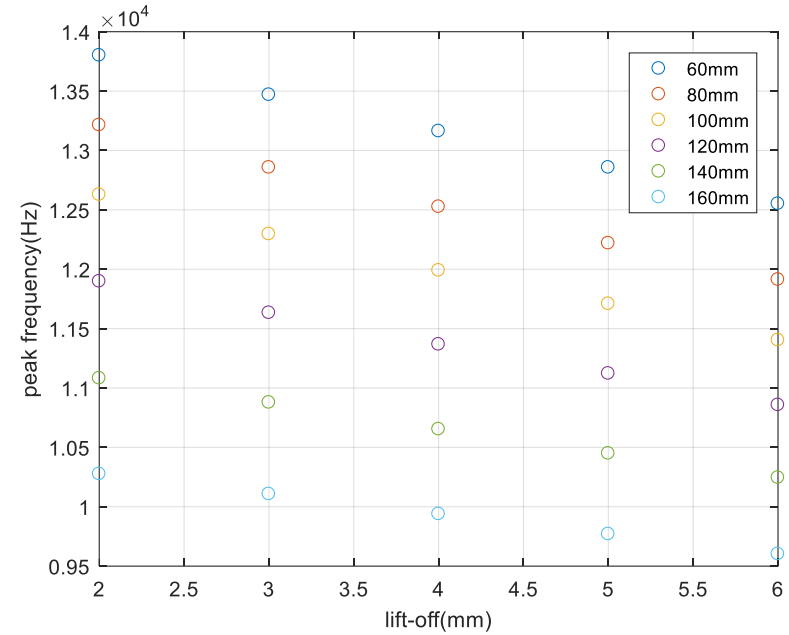

Fig.13. Comparisons of measured peak frequencies with thickness of 1-mm stainless steel ball at a range of lift-offs

TABLE III

RADIUS MEASUREMENTS FOR DIFFERENT RADIUS AND LIFT-OFFS

\begin{tabular}{llllll}
\hline \hline $\begin{array}{l}\text { Lift-off } \\
\text { point1 } \\
(\mathrm{mm})\end{array}$ & $\begin{array}{l}\text { Lift-off } \\
\text { point2 } \\
(\mathrm{mm})\end{array}$ & $\begin{array}{l}\text { Actual } \\
\text { radius } \\
(\mathrm{mm})\end{array}$ & $\begin{array}{l}\text { Slope of } \\
\text { peak- } \\
\text { frequency } \\
\text { versus } \\
\text { lift-off }\end{array}$ & $\begin{array}{l}\text { Radius } \\
\text { calculated } \\
\text { from } \\
\text { original as } \\
\text { calculated } \\
(\mathrm{mm})\end{array}$ & $\begin{array}{l}\text { relative } \\
\text { error }\end{array}$ \\
\hline 2 & 3 & 140 & -204 & 142.8 & $2.0 \%$ \\
3 & 4 & 140 & -225 & 141.7 & $1.2 \%$ \\
4 & 5 & 140 & -204 & 140.8 & $0.5 \%$ \\
2 & 3 & 160 & -169 & 159.6 & $0.8 \%$ \\
3 & 4 & 160 & -168 & 158.7 & $0.8 \%$ \\
4 & 5 & 160 & -169 & 158.6 & $0.8 \%$ \\
\hline
\end{tabular}

\section{CONCLUSIONS}

In this paper, it is found that the peak frequency feature of inductance spectrum is linear to the lift-off spacing between the centre of coil and ball. Besides, the slope of peak frequencies versus lift-offs is linked to the radius of ball. Thus, the proposed approach is using the sensor probe to measure the slope of peak frequencies under different lift-off from receive coil. Then applying the radius calculation formula which is proposed in paper to obtain the radius of metallic balls. The results from the experiments show that the radii of samples can be predicted accurately within the error of $2 \%$. The shortcoming of this experimental design is that the sensor needs to be measured twice to get a set of experimental data values. Therefore, a triple-coil sensor will be designed in future, in which three coils (two excitation coils and one receiving coil) with same radii are co-axially arranged in different lift-off planes. Based on this sensor setup, the measurement method applied in this paper can be optimized. By utilizing two transmitter-receiver combinations and the proposed algorithm, the radius of metallic ball can be obtained by measuring once. 


\section{ACKNOWLEDGEMENT}

This work was supported by [UK Engineering and Physical Sciences Research Council (EPSRC)] [grant number: EP/P027237/1] [title: Real-time In-line Microstructural Engineering (RIME)].

\section{REFERENCES}

[1]. Takagi T, Miya K. ECT round-robin test for steam generator tube. J Jpn Soc Appl Electromagn Mech 2000;8:121-8.

[2]. Moulder J, Uzal CE, Rose JH. Thickness and conductivity of metallic layers from eddy current measurements. Rev Sci Instrum 1992;63:3455-65.

[3]. Nonaka Y. A double coil method for simultaneously measuring the resistivity, permeability, and thickness of a moving metal sheet. IEEE Trans Instrum Meas 1996;45:478-82.

[4]. Placko D, Clergeot H, Santander E. Physical modelling of an eddy current sensor designed for real time distance and thickness measurement in galvanization industry. IEEE Trans Magn 1989;25:2861-3.

[5]. Sethuraman A, Rose JH. "Rapid inversion of eddy current data for conductivity and thickness of metal coatings." J Nondestructive Eval 1995;14:39-46.

[6]. R. K. Amineh, et al, "Using AC field measurement data at an arbitrary lift-off distance to size long surface-breaking cracks in ferrous metals," NDT \& E Int., vol. 41, no. 3, pp. 169-177, 2008.

[7]. R. K. Amineh, et al, "Removal of probe lift-off effects on crack detection and sizing in metals by the AC field measurement technique," IEEE Trans. Magnetics, vol. 44, no. 8, pp. 2066-2073, 2008.

[8]. G. Y. Tian and A. Sophian, "Reduction of lift-off effects for pulsed eddy current NDT," NDT \& E International, vol. 38, no. 4, pp. 319-324, 2005.

[9]. Yang, B. Li and Y. Wang, "Reduction of lift-off effect for pulsed eddy current NDT based on sensor design and frequency spectrum analysis", Nondestructive Testing and Evaluation, vol. 25, no. 1, pp. 77-89, 2010.

[10]. Y. Wang, M. Fan, B. Cao, B. Ye, and D. Wen, "Measurement of coating thickness using lift-off point of intersection features from pulsed eddy current signals," NDT \& E International, vol. 116, pp. 102333, 2020.

[11]. C. S. Angani et al. "Lift-off point of intersection feature in transient eddy current oscillations method to detect thickness variation in stainless steel," IEEE Transactions on Magnetics, vol. 52, no. 6, pp. 1-8, 2016.

[12]. A. I. Nikitin," Interaction of eddy current transducers with layer construction shells of curved form and instruments for measuring the dimensions of these shells(Review)," The Soviet Journal of Nondestructive Testing, vol. 16, no. 11, pp.775-792, 1980

[13]. J. Kral, R. Smid, H. M. G. Ramos, and A. L. Ribeiro, "The lift-off effect in eddy currents on thickness modeling and measurement," IEEE Trans. Instrum. Meas., vol. 62, no. 7, pp.2043-2049, 2013

[14]. W. Yin and K. Xu, "A novel triple-coil electromagnetic sensor for thickness measurement immune to lift-off variations," IEEE Trans. Instrum. Meas., vol. 65 , no. 1, pp.164-169, 2016

[15]. W. Yin and A. J. Peyton, "Thickness measurement of metallic plates with an electromagnetic sensor using phase signature analysis," IEEE Trans. Instrum. Meas., vol. 57, no. 8, pp.1803-1807, 2008

[16]. M. Lu, L. Yin, A. J. Peyton, and W. Yin, "A novel compensation algorithm for thickness measurement immune to lift-off variations using eddy current method," IEEE Trans. Instrum. Meas., vol. 65, no. 12, pp.2773-2779, 2016

[17]. G. Y. Tian and A. Sophian, "Reduction of lift-off effects for pulsed eddy current NDT," NDT \& E International, vol. 38, no. 4, pp. 319-324, 2005.

[18]. B. A. Abu-Nabah, "Reduction of lift-off effect in high-frequency apparent eddy current conductivity spectroscopy," Measurement Science and Technology, vol. 28, no. 5, pp. 055-107, 2017.

[19]. J. C. Arez, H. M. G. Ramos, and A. L. Ribeiro, "lift-off insensitive thickness measurement of aluminum plates using harmonic eddy current excitation and a GMR sensor," Measurement., vol. 45, no. 9, pp.2246-2253, 2012

[20]. D. F. He and M Yoshizawa, "Dual-frequency eddy current NDE based on high-Tc rf SQUID," Physica C: Superconductivity, vol. 383, no. 3, pp.223226, 2002

[21]. W. Yin and A.J. Peyton, "Thickness measurement of non-magnetic plates using multi-frequency eddy current sensors," NDT \& E International, vol. 40, no. 1, pp. 43-48, 2007.

[22]. M. Lu, R. Huang, W. Yin, Q. Zhao, and A. Peyton, "Measurement of permeability for ferrous metallic plates using a novel lift-off compensation technique on phase signature," IEEE Sensors Journal, vol. 19, no. 17, pp. 7440-7446, 1 Sept.1, 2019.

[23]. M. Lu, et al. "Determination of the magnetic permeability, electrical conductivity, and thickness of ferrite metallic plates using a multifrequency electromagnetic sensing system," IEEE Transactions on Industrial Informatics, vol. 15, pp. 4111-4119, 2019.

[24]. J.R.S. Avila, M. Lu et al. "Accurate measurements of plate thickness with variable lift-off using a combined inductive and capacitive sensor," NDT \& E International, vol. 110, pp. 102202, 2020.

[25]. W. Yin, S. J. Dickinson, and A. Peyton, "Imaging the continuous conductivity profile within layered metal structures using inductance spectroscopy,” IEEE Sensors Journal, vol. 5, no. 2, pp. 161-166, 2005.

[26]. M. Lu, H. Xu, W. Zhu, L. Yin et al. "Conductivity Lift-off Invariance and measurement of permeability for ferrite metallic plates," NDT \& E International, vol. 95, pp. 36-44, Apr. 2018.

[27]. M. Lu, L. Yin, A. J. Peyton and W. Yin, "A novel compensation algorithm for thickness measurement immune to lift-off variations using eddy current method," IEEE Transactions on Instrumentation and Measurement, vol. 65, no. 12, pp. 2773-2779, Dec. 2016.

[28]. M. Lu, X. Meng, W. Yin, Z. Qu, F. Wu, J. Tang, et al., "Thickness measurement of non-magnetic steel plates using a novel planar triple-coil sensor," NDT \& E International, vol. 107, 2019.

[29]. R. Huang, M. Lu, A. Peyton, and W. Yin, "Thickness measurement of metallic plates with finite planar dimension using eddy current method," IEEE Transactions on Instrumentation and Measurement, early access, 2020. Doi:10.1109/TIM.2020.2987413.

[30]. M. Lu et al. "Measurement of ferromagnetic slabs permeability based on a novel planar triple-coil sensor," IEEE Sensors J., vol. 20, no. 6, pp. 2904-2910, 2020.

[31]. M. Lu, W. Zhu, L. Yin, A. J. Peyton, W. Yin, and Z. Qu, "Reducing the lift-off effect on permeability measurement for magnetic plates from multifrequency induction data," IEEE Transactions on Instrumentation and Measurement, vol. 67, no. 1, pp. 167-174, Jan. 2018.

[32]. M. Fan et al., "Pulsed eddy current thickness measurement using phase features immune to liftoff effect," NDT \& E International, vol. 86, pp. 123-131, 2017.

[33]. M. Lu, L. Chen, X. Meng, R. Huang, A. Peyton and W. Yin, "Thickness measurement of metallic film based on a high-frequency feature of triple-coil electromagnetic eddy current sensor," IEEE Transactions on Instrumentation and Measurement, pp. 1-1, 2020.

[34]. Lu, M, Meng, X, Huang, R, Chen, L, Peyton, A and Yin, W, "Lift-off tolerant pancake eddy-current sensor for the thickness and spacing measurement of non-magnetic plates", IEEE Transactions on Instrumentation and Measurement. pp. 1-1, 2020.

[35]. Lu, M, Meng, X, Huang, R, Chen, L, Peyton, A and Yin, W 2020, "Inversion of distance and magnetic permeability based on material-independent and liftoff insensitive algorithms using eddy current sensor", IEEE Transactions on Instrumentation and Measurement. pp. 1-1, 2020.

[36]. Lu, M, Meng, X, Huang, R, Chen, L, Peyton, A and Yin, W 2020, "Measuring lift-off distance and electromagnetic property of metal using dual-frequency linearity feature", IEEE Transactions on Instrumentation and Measurement. pp. 1-1, 2020.

[37]. T.P. Theodoulidis, "Analytical modeling of wobble in eddy current tube testing with bobbin coils," Journal of Research in Nondestructive Evaluation, 14 (2), pp. 111-126, 2002.

[38]. M. Lu, W. Zhu, L. Yin, A.J. Peyton, W. Yin, and Z. Qu, "Reducing the LiftOff Effect on Permeability Measurement for Magnetic Plates from Multifrequency Induction Data." IEEE Trans. Instrum. Meas., vol. 67, no. 1, pp. 167-174, 2018.

[39]. M. Lu, R. Huang, W. Yin, Q. Zhao and A. Peyton, "Measurement of Permeability for Ferrous Metallic Plates Using a Novel Lift-Off Compensation Technique on Phase Signature," IEEE Sensors Journal, vol. 19, no. 17, pp. 7440-7446, 1 Sept.1, 2019.

[40]. T. Theodoulidis and E. Kriezis, "Coil impedance due to a sphere of arbitrary radial conductivity and permeability profiles", IEEE Transactions on Magnetics, vol. 38, no. 3, pp. 1452-1460, 2002.

[41]. R. Huang, M. Lu, A. Peyton, and W. Yin, "Thickness measurement of metallic plates with finite planar dimension using eddy current method," IEEE Transactions on Instrumentation and Measurement, early access, 2020. Doi:10.1109/TIM.2020.2987413.

[42]. M. Lu, et al. "Acceleration of frequency sweeping in eddy-current computation," IEEE Transactions on Magnetics, vol. 53, pp. 1-8, 2017. 
[43]. W. Yin et al, "Measurement of permeability and ferrite/austenite phase fraction using a multi-frequency electromagnetic sensor," NDT \& E International, vol. 42, no. 1, pp. 64-68, 2009.

[44]. M. Lu, H. Xu, W. Zhu, L. Yin et al. "Conductivity Lift-off Invariance and measurement of permeability for ferrite metallic plates," NDT \& E International, vol. 95, pp. 36-44, Apr. 2018.

[45]. W. Yin et al. "Permeability invariance phenomenon and measurement of electrical conductivity for ferrite metallic plates," Insight-NonDestructive Testing and Condition Monitoring, vol. 61, pp. 472-479, 2019.

[46]. M. Lu, W. Zhu, L. Yin, A. J. Peyton, W. Yin, and Z. Qu, "Reducing the lift-off effect on permeability measurement for magnetic plates from multifrequency induction data," IEEE Transactions on Instrumentation and Measurement, vol. 67, no. 1, pp. 167-174, Jan. 2018.

[47]. M. Lu, L. Yin, A. J. Peyton and W. Yin, "A novel compensation algorithm for thickness measurement immune to lift-off variations using eddy current method," IEEE Transactions on Instrumentation and Measurement, vol. 65, no. 12, pp. 2773-2779, Dec. 2016

[48]. M. Lu, R. Huang, W. Yin, Q. Zhao, and A. Peyton, "Measurement of permeability for ferrous metallic plates using a novel lift-off compensation technique on phase signature," IEEE Sensors Journal, vol. 19, no. 17, pp. 7440-7446, 1 Sept.1, 2019.

[49]. M. Lu et al. "Determining the magnetic permeability of ferrite steel strip by a custom inversion method," Proc. 12th ECNDT, pp. 1-8, 2018.

[50]. M. Lu, et al. "Determination of the magnetic permeability, electrical conductivity, and thickness of ferrite metallic plates using a multifrequency electromagnetic sensing system," IEEE Transactions on Industrial Informatics, vol. 15, pp. 4111-4119, 2019.

[51]. J.R.S. Avila, M. Lu et al. "Accurate measurements of plate thickness with variable lift-off using a combined inductive and capacitive sensor," NDT \& E International, vol. 110, pp. 102202, 2020.

[52]. M. Lu, R. Huang, W. Yin, Q. Zhao, and A. Peyton, "Measurement of permeability for ferrous metallic plates using a novel lift-off compensation technique on phase signature," IEEE Sensors Journal, vol. 19, no. 17, pp. 7440-7446, 1 Sept.1, 2019

[53]. M. Lu, X. Meng, W. Yin, Z. Qu, F. Wu, J. Tang, et al., "Thickness measurement of non-magnetic steel plates using a novel planar triple-coil sensor," NDT \& E International, vol. 107, 2019.

[54]. M. Lu et al. "Measurement of ferromagnetic slabs permeability based on a novel planar triple-coil sensor," IEEE Sensors J., vol. 20, no. 6, pp. 2904-2910, 2020.

[55]. M. Lu, et al, "Determination of surface crack orientation based on thinskin regime using triple-coil drive-pickup eddy-current sensor," IEEE Transactions on Instrumentation and Measurement, vol. 70, 2020.

[56]. J.R.S. Avila, M. Lu et al. "Accurate measurements of plate thickness with variable lift-off using a combined inductive and capacitive sensor," NDT \& E International, vol. 110, pp. 102202, 2020.

[57]. M. Lu, L. Yin, A. J. Peyton and W. Yin, "A novel compensation algorithm for thickness measurement immune to lift-off variations using eddy current method," IEEE Transactions on Instrumentation and Measurement, vol. 65, no. 12, pp. 2773-2779, Dec. 2016.

[58]. M. Lu, X. Meng, W. Yin, Z. Qu, F. Wu, J. Tang, et al., "Thickness measurement of non-magnetic steel plates using a novel planar triplecoil sensor," NDT \& E International, vol. 107, 2019.

[59]. R. Huang, M. Lu, A. Peyton, and W. Yin, "Thickness measurement of metallic plates with finite planar dimension using eddy current method," IEEE Transactions on Instrumentation and Measurement, early access, 2020. Doi:10.1109/TIM.2020.2987413.

[60]. M. Lu et al. "Measurement of ferromagnetic slabs permeability based on a novel planar triple-coil sensor," IEEE Sensors J., vol. 20, no. 6, pp. 2904-2910, 2020

[61]. M. Lu, W. Zhu, L. Yin, A. J. Peyton, W. Yin, and Z. Qu, "Reducing the lift-off effect on permeability measurement for magnetic plates from multifrequency induction data," IEEE Transactions on Instrumentation and Measurement, vol. 67, no. 1, pp. 167-174, Jan. 2018.

[62]. R. Huang, M. Lu et al, "Measurement of the radius of metallic plates based on a novel finite region eigenfunction expansion (FREE) method," IEEE Sensors Journal, vol. 20, pp. 15099 - 15106, 2020. Doi: 10.1109/JSEN.2020.3009443

[63]. R. Huang, M. Lu et al, "Measuring co-axial hole size of finite-size metallic disk based on a dual-constraint integration feature using multifrequency eddy current testing," IEEE Transactions on Instrumentation and Measurement, vol. 70, pp. 1-7, 2020. Doi 10.1109/TIM.2020.3026762.

[64]. M. Lu et al, "Thickness Measurement of Metallic Film Based on a High-Frequency Feature of Triple-Coil Electromagnetic Eddy Current Sensor," IEEE Transactions on Instrumentation and Measurement, vol. 70, pp. 1-8, 2020. Doi: 10.1109/TIM.2020.3027929.

[65]. M. Lu et al, "Measuring Lift-Off Distance and Electromagnetic Property of Metal Using Dual-Frequency Linearity Feature," IEEE Transactions on Instrumentation and Measurement, vol. 70, pp. 1-9, 2020. Doi: 10.1109/TIM.2020.3029348.

[66]. M. Lu et al, "Lift-off tolerant pancake eddy-current sensor for the thickness and spacing measurement of non-magnetic plates," IEEE Transactions on Instrumentation and Measurement, early access, 2020. Doi: 10.1109/TIM.2020.3033377.

[67]. M. Lu et al, "Inversion of distance and magnetic permeability based on material-independent and lift-off insensitive algorithms using eddy current sensor," IEEE Transactions on Instrumentation and Measurement, early access, 2020. Doi: 10.1109/TIM.2020.3036099.

[68]. W. Yin et al, "Measurements of Thickness for Metallic Plates With CoAxial Holes Using a Novel Analytical Method With the Modified Integration Range," IEEE Access, vol. 8, pp. 198301 - 198306, 2020.

[69]. X. Meng, M. Lu et al, "Inversion of lift-off distance and thickness for non-magnetic metal using eddy current testing," IEEE Transactions on Instrumentation and Measurement, vol. 70, 2020. Doi: 10.1109/TIM.2020.3038289

[70]. M. Lu, X. Meng, et al, "Determination of surface crack orientation based on thin-skin regime using triple-coil drive-pickup eddy-current sensor," IEEE Transactions on Instrumentation and Measurement, early access, 2020. Doi: 10.1109/TIM.2020.3044729.

[71]. Yin, W.; Tang, J.; Lu, M.; et al. An equivalent-effect phenomenon in eddy current non-destructive testing of thin structures. IEEE Access, 2019, 7, pp. 70296-70307.

[72]. Lu, M.; et al. Determination of Surface Crack Orientation Based on Thin-Skin Regime Using Triple-Coil Drive-Pickup Eddy-Current Sensor. IEEE Transactions on Instrumentation and Measurement, 2020, 70, pp. 1-9. DOI: 10.1109/TIM.2020.3044729

[73]. M. Lu, et al., "Prediction of the asymptotical magnetic polarization tensors for cylindrical samples using the boundary element method," In 2015 IEEE Sensors Applications Symposium (SAS), pp. 1-4. IEEE, 2015.

[74]. R. Huang, M. Lu, A. Peyton, and W. Yin, "A novel perturbed matrix inversion based method for the acceleration of finite element analysis in crack-scanning eddy current NDT," IEEE Access, vol. 8, pp. 1243812444,2020

[75]. J. Tang et al., "A Novel Efficient FEM Thin Shell Model for BioImpedance Analysis," Biosensors, vol. 10, no. 6, pp. 69, 2020.

[76]. L. Chen, et al., "Textile Based Capacitive Sensor for Physical Rehabilitation via Surface Topological Modification," ACS Nano, vol. 14, no. 7, pp. 8191-8201, 2020. DOI: 10.1021/acsnano.0c01643

[77]. Z. Jin, et al., "Methods of Controlling Lift-off in Conductivity Invariance Phenomenon for Eddy Current Testing," IEEE ACCESS, vol. 8, pp. 2169-3536, 2020. DOI: 10.1109/ACCESS.2020.3007216.

[78]. J. Tang, et al., "Effect of frozen-thaw injury on cell membrane and bioimpedance," In 2020 IEEE International Instrumentation and Measurement Technology Conference (I2MTC), pp. 1-6. IEEE, 2020

[79]. J. Tang, et al., "Bio-impedance spectroscopy for frozen-thaw of biosamples: Non-contact inductive measurement and finite element (FE) based cell modelling," Journal of Food Engineering, vol. 272, pp. 109784, 2020.

[80]. H. Xu et al., "Imaging a weld cross-section using a novel frequency feature in multi-frequency eddy current testing," Insight-NonDestructive Testing and Condition Monitoring, vol. 61, no. 12, pp. 738 - 743, 2019.

[81]. Y. Xie et al., "Novel Wearable Sensors for Biomechanical Movement Monitoring Based on Electromagnetic Sensing Techniques," IEEE Sensors Journal, vol. 20, no. 2, 2020. DOI: 10.1109/JSEN.2019.2943487

[82]. W. Yin et al., "Permeability invariance phenomenon and measurement of electrical conductivity for ferrite metallic plates," Insight-NonDestructive Testing and Condition Monitoring, vol. 61, no. 8, pp. 472 479, 2019. 
[83]. M. Lu et al., "A model for the triboelectric nanogenerator with inductive load and its energy boost potential," Nano Energy, vol. 63, pp. 103883, 2019.

[84]. M. Lu et al., "Forward solver for deep earth exploration and induction logging using custom built Edge-Element FEM technique," Acta Geologica Sinica, vol. 93, pp. 302-304, 2019.

[85]. L. Chen et al., "Whole System Design of Wearable Magnetic Induction Sensor for Physical Rehabilitation," Advanced Intelligent Systems, vol. 1, no. 1, pp. 1900037, 2019.

[86]. Y. X et al., "A self-powered radio frequency (RF) transmission system based on the combination of triboelectric nanogenerator (TENG) and piezoelectric element for disaster rescue/relief," Nano Energy, vol. 54, pp. 331-340, 2018.

[87]. W. Yin et al., "Custom edge-element FEM solver and its application to eddy-current simulation of realistic $2 \mathrm{M}$-element human brain phantom," Bioelectromagnetics, vol. 39, no. 8, pp. 604-616, 2018.

[88]. L. Yin et al., "Detection of corrosion pits based on an analytically optimised eddy current sensor," Insight-Non-Destructive Testing and Condition Monitoring, vol. 60, no. 10, pp. 561-567, 2018.

[89]. W. Yin et al., "Acceleration of eddy current computation for scanning probes," Insight-Non-Destructive Testing and Condition Monitoring, vol. 60, no. 10, pp. 547-555, 2018.

[90]. W. Zhou et al., "Three-dimensional electromagnetic mixing models for dual-phase steel microstructures," Applied Sciences, vol. 8, no. 4, pp. 547-555, 2018.

[91]. M. Lu, et al., "Determining the magnetic permeability of ferrite steel strip by a custom inversion method," In Proc. 12th ECNDT, pp. 1-8. 2018.

[92]. J. Tang, et al., "Cellular structure analysis based on magnetic induction finite element method simulations and measurements," bioRxiv, pp. 275271, 2018. DOI: $10.1101 / 275271$

[93]. J.R.S. Avila, et al., "A novel dual modality sensor with sensitivities to permittivity, conductivity, and permeability," IEEE Sensors Journal, vol. 18, no. 1, pp. 356-362, 2017.

[94]. T. Yang, et al., "Level measurement for saline with a small surface area using high frequency electromagnetic sensing technique," Measurement, vol. 101, pp. 118-125, 2017.

[95]. X. Meng, et al, "Evaluation of coating thickness using lift-off insensitivity of eddy current sensor," Sensors, vol. 21, no. 2, pp. 419, 2021.

[96]. Meng, X.; Lu, M.; Yin, W.; Bennecer, A.; Kirk, K.J. Evaluation of Coating Thickness Using Lift-Off Insensitivity of Eddy Current Sensor. Sensors 2021, 21, 419. https://doi.org/10.3390/s21020419

[97]. M. Lu, et al., " Thickness measurement of circular metallic film using single-frequency eddy current sensor," NDT \& E International, vol. 119, pp. 102420, 2021.

[98]. Jin, Zhongwen, et al. "Boundary-element analysis of magnetic polarization tensor for metallic cylinder." IEEE Access 9 (2021): 63250-63256.

[99]. Lu, M.; Meng, X.; Huang, R.; Chen, L.; Peyton, A.; Yin, W. Lift-off Invariant Inductance of Steels in Multi-Frequency Eddy-Current Testing. NDT\&E International, vol. 121, p. 102458, 2021.

\section{APPENDIX}

Case 1: $\sigma_{t+1} \neq 0$ and $\sigma_{t} \neq 0$

$T_{11}(t+1, t)=\left[\frac{i_{n}\left(a_{t} b_{t}\right)}{\mu_{t+1}}\left[k_{n}\left(a_{t+1} b_{t}\right)+\left(a_{t+1} b_{t}\right) k_{n}^{\prime}\left(a_{t+1} b_{t}\right)\right]\right.$

$\left.-\frac{k_{n}\left(a_{t+1} b_{t}\right)}{\mu_{t}}\left[i_{n}\left(a_{t} b_{t}\right)+\left(a_{t} b_{t}\right) i_{n}^{\prime}\left(a_{t} b_{t}\right)\right]\right] / D E N$

$T_{12}(t+1, t)=\left[\frac{k_{n}\left(a_{t} b_{t}\right)}{\mu_{t+1}}\left[k_{n}\left(a_{t+1} b_{t}\right)+\left(a_{t+1} b_{t}\right) k_{n}^{\prime}\left(a_{t+1} b_{t}\right)\right]\right.$

$\left.-\frac{k_{n}\left(a_{t+1} b_{t}\right)}{\mu_{t}}\left[k_{n}\left(a_{t} b_{t}\right)+\left(a_{t} b_{t}\right) k_{n}^{\prime}\left(a_{t} b_{t}\right)\right]\right] / D E N$

$$
\begin{aligned}
& T_{21}(t+1, t)=\left[\frac{i_{n}\left(a_{t+1} b_{t}\right)}{\mu_{t}}\left[i_{n}\left(a_{t} b_{t}\right)+\left(a_{t} b_{t}\right) i_{n}^{\prime}\left(a_{t} b_{t}\right)\right]\right. \\
& \left.-\frac{i_{n}\left(a_{t} b_{t}\right)}{\mu_{t+1}}\left[i_{n}\left(a_{t+1} b_{t}\right)+\left(a_{t+1} b_{t}\right) i_{n}^{\prime}\left(a_{t+1} b_{t}\right)\right]\right] / D E N \\
& T_{22}(t+1, t)=\left[\frac{i_{n}\left(a_{t+1} b_{t}\right)}{\mu_{t}}\left[k_{n}\left(a_{t} b_{t}\right)+\left(a_{t} b_{t}\right) k_{n}^{\prime}\left(a_{t} b_{t}\right)\right]\right. \\
& \left.-\frac{k_{n}\left(a_{t} b_{t}\right)}{\mu_{t+1}}\left[i_{n}\left(a_{t+1} b_{t}\right)+\left(a_{t+1} b_{t}\right) i_{n}^{\prime}\left(a_{t+1} b_{t}\right)\right]\right] / D E N \\
& D E N=\frac{a_{t+1} b_{t}}{\mu_{t+1}}\left[i_{n}\left(a_{t+1} b_{t}\right) k_{n}^{\prime}\left(a_{t+1} b_{t}\right)-i_{n}^{\prime}\left(a_{t+1} b_{t}\right) k_{n}\left(a_{t+1} b_{t}\right)\right]
\end{aligned}
$$

Case 2: $\sigma_{t+1} \neq 0$ and $\sigma_{t}=0$

$T_{11}(t+1, t)=\frac{b_{t}^{n}}{\mu_{t+1}}\left[k_{n}\left(a_{t+1} b_{t}\right)\left[1-(n+1) \mu_{t+1}\right]\right.$

$\left.+a_{t+1} b_{t} k_{n}^{\prime}\left(a_{t+1} b_{t}\right)\right] / D E N$

$T_{12}(t+1, t)=\frac{1}{b_{t}^{n+1} \mu_{t+1}}\left[k_{n}\left(a_{t+1} b_{t}\right)\left(1+n \mu_{t+1}\right)\right.$

$\left.+a_{t+1} b_{t} k_{n}^{\prime}\left(a_{t+1} b_{t}\right)\right] / D E N$

$T_{21}(t+1, t)=-\frac{b_{t}^{n}}{\mu_{t+1}}\left[i_{n}\left(a_{t+1} b_{t}\right)\left[1-(n+1) \mu_{t+1}\right]\right.$

$\left.+a_{t+1} b_{t} i_{n}^{\prime}\left(a_{t+1} b_{t}\right)\right] / D E N$

$T_{22}(t+1, t)=-\frac{1}{b_{t}^{n+1} \mu_{t+1}}\left[i_{n}\left(a_{t+1} b_{t}\right)\left(1+n \mu_{t+1}\right)\right.$

$\left.+a_{t+1} b_{t} i_{n}^{\prime}\left(a_{t+1} b_{t}\right)\right] / D E N$

Case 3: $\sigma_{t+1}=0$ and $\sigma_{t} \neq 0$

$T_{11}(t+1, t)=\frac{1}{b_{t}^{n} \mu_{t}}\left[i_{n}\left(a_{t} b_{t}\right)\left[1+n \mu_{t}\right]\right.$

$\left.+a_{t} b_{t} i_{n}^{\prime}\left(a_{t} b_{t}\right)\right] /(2 n+1)$

$T_{12}(t+1, t)=\frac{1}{b_{t}^{n} \mu_{t}}\left[k_{n}\left(a_{t} b_{t}\right)\left[1+n \mu_{t}\right]\right.$

$\left.+a_{t} b_{t} k_{n}^{\prime}\left(a_{t} b_{t}\right)\right] /(2 n+1)$

(30)

$T_{21}(t+1, t)=-\frac{b_{t}^{n+1}}{\mu_{t}}\left[i_{n}\left(a_{t} b_{t}\right)\left[1-(n+1) \mu_{t}\right]\right.$

$\left.+a_{t} b_{t} i_{n}^{\prime}\left(a_{t} b_{t}\right)\right] /(2 n+1)$

$T_{22}(t+1, t)=-\frac{b_{t}^{n+1}}{\mu_{t}}\left[k_{n}\left(a_{t} b_{t}\right)\left[1-(n+1) \mu_{t}\right]\right.$

$\left.+a_{t} b_{t} k_{n}^{\prime}\left(a_{t} b_{t}\right)\right] /(2 n+1)$ 